\title{
Thick Transparent Nanoparticle-Based Mesoporous Silica Monolithic Slabs for Thermally Insulating Window Materials
}

\section{- Supporting Information -}

Michal Marszewski, ${ }_{1}$ Sophia C. King, ${ }^{2}$ Yan Yan, ${ }^{2}$ Tiphaine Galy, ${ }^{1}$ Man Li, ${ }^{1}$ Ali Dashti, ${ }^{1}$ Danielle M. Butts, ${ }^{3}$ Joon Sang Kang, ${ }^{1}$ Patricia E. McNeil, ${ }^{3}$ Esther Lan, ${ }^{3}$ Bruce Dunn, ${ }^{3,4}$ Yongjie Hu, ${ }^{1}$ Sarah H. Tolbert, ${ }^{2,3,4}$ Laurent Pilon ${ }^{1,4,5^{*}}$

${ }^{1}$ Mechanical and Aerospace Engineering Department, University of California, Los Angeles, Los Angeles, California 90095, USA

${ }^{2}$ Department of Chemistry and Biochemistry, University of California, Los Angeles, Los Angeles, California 90095, USA

${ }^{3}$ Department of Materials Science and Engineering, University of California, Los Angeles, Los Angeles, California 90095, USA

${ }^{4}$ California NanoSystems Institute, University of California, Los Angeles, Los Angeles, California 90095, USA

${ }^{5}$ Institute of the Environment and Sustainability, University of California, Los Angeles, Los Angeles, California 90095, USA

*E-mail: pilon@seas.ucla.edu

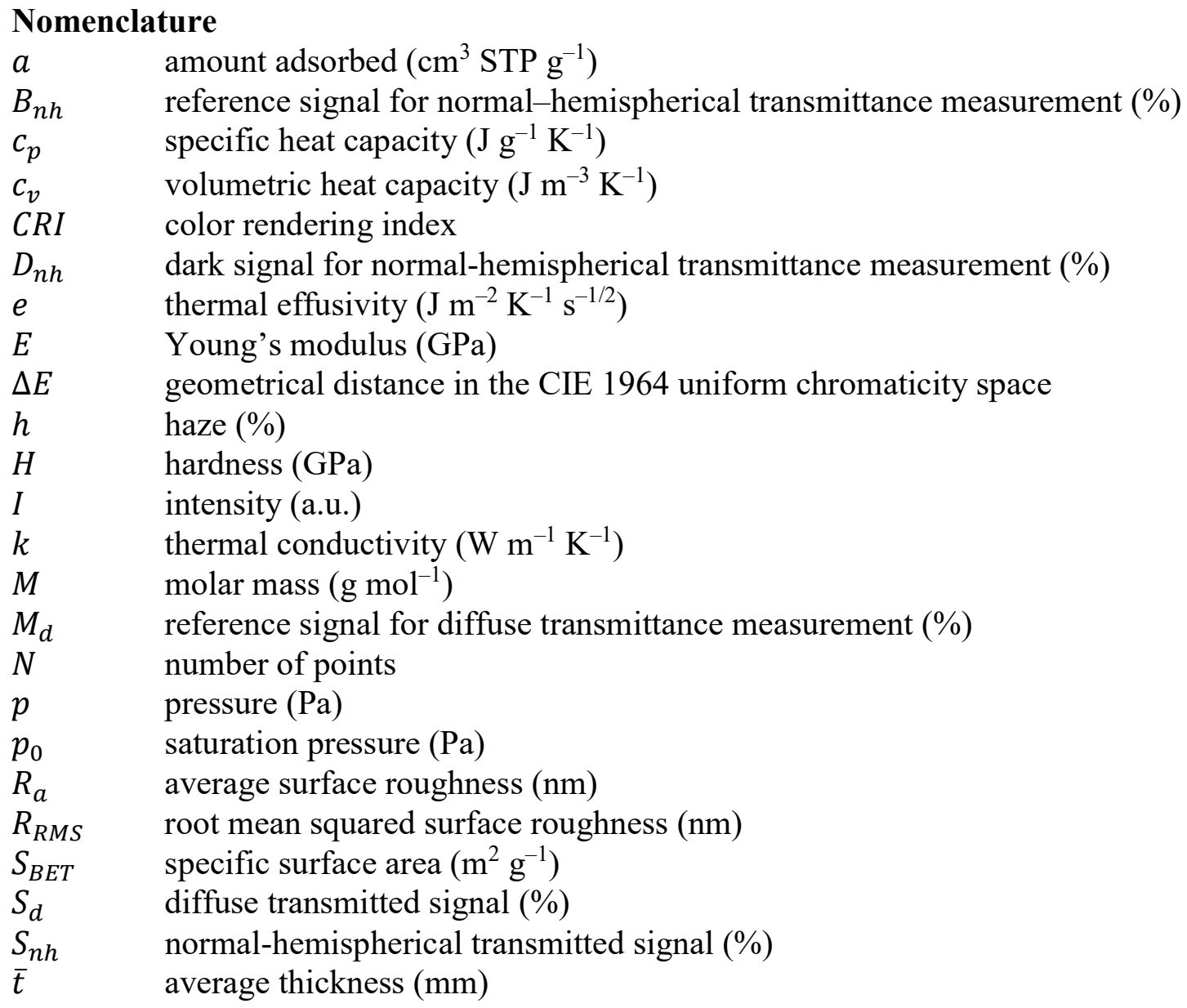




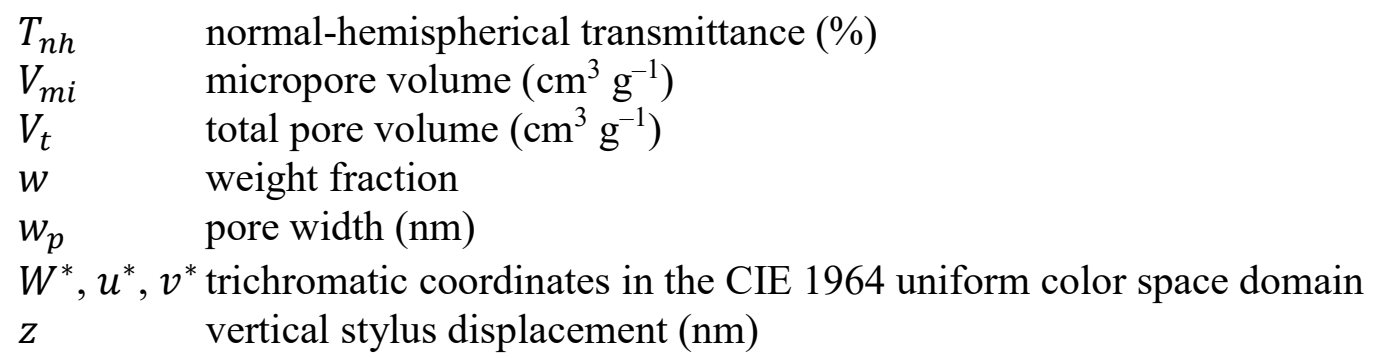

Greek symbols

$\alpha_{s} \quad$ reduced adsorption

$\lambda \quad$ wavelength (nm)

$\rho \quad$ density $\left(\mathrm{g} \mathrm{cm}^{-3}\right)$

$\sigma \quad$ surface density of surface groups $\left(\mathrm{mol} \mathrm{m}^{-2}\right)$

$\phi \quad$ porosity or volume fraction

Subscripts and superscripts

ave refers to average

eff refers to effective

exp refers to experimental

fit refers to values retrieved from fit

$\mathrm{H}_{2} \mathrm{O} \quad$ refers to water

ref refers to reference material

$\mathrm{OH} \quad$ refers to surface hydroxyl groups

$\mathrm{SiO}_{2} \quad$ refers to silica

\section{S1. Materials and methods}

Chemicals. Colloidal solution of silica nanoparticles Nalco 2326 (15 wt $\%$ in water, $\mathrm{NH}_{3}$ stabilized, lot number BP7J1239A1) was purchased from Nalco Chemical Company (Naperville, IL, USA). Perfluoropolyether oils Krytox GPL 100, GPL 104, and GPL 106 with general formula $\left[\mathrm{CF}\left(\mathrm{CF}_{3}\right) \mathrm{CF}_{2} \mathrm{O}\right]_{n}$ where $n=10-60$, used as the liquid substrates, were purchased from MillerStephenson Chemical Company Inc. (Danbury, CT, USA). All chemicals were used as received without further purification.

Material synthesis. The exact synthesis of nanoparticle-based mesoporous silica slabs on PFC was as follows: between 5 and $30 \mathrm{~mL}$ of the colloidal solution of silica nanoparticles was placed in a PTFE mold with PFC liquid (either Krytox GLP 100, GPL 104 or GLP 106) covering the bottom 
surface in a $1-3 \mathrm{~mm}$ thick layer. Water was slowly removed by evaporation resulting in gelation and aging of nanoparticle-based slabs in the shape of the mold. The slabs were then slowly dried to remove all water without cracking leading to mesoporous silica slabs. The drying rate was controlled via (i) the mold's opening size, (ii) temperature, and (iii) surrounding relative humidity. Here, the samples were dried either (i) in ambient air at room temperature or in a convection oven at $25{ }^{\circ} \mathrm{C}$, with a mold's opening corresponding to $0-10 \%$ of the mold surface area, (ii) in a convection oven at $40{ }^{\circ} \mathrm{C}$ with a mold's opening completely covered, or (iii) in a humidity chamber at room temperature and relative humidity of $50-80 \%$. The resulting mesoporous silica slabs were calcined in oxygen at $400{ }^{\circ} \mathrm{C}$ for $2 \mathrm{~h}$ using a $5{ }^{\circ} \mathrm{C} \mathrm{min}^{-1}$ temperature ramp to remove any $\mathrm{NH}_{3}$ and PFC residues.

The synthesis of mesoporous silica slabs on a solid PTFE substrate was the same as that described above except that the colloidal solution of silica nanoparticles was placed in an empty PTFE mold without PFC liquid. In addition, the mesoporous slabs prepared on PTFE were analyzed as synthesized, i.e., without calcination.

\section{S2. Characterization methods}

Structural Characterization. The average thickness $\bar{t}$ (in $\mathrm{mm}$ ) of nanoparticle-based mesoporous silica slabs was estimated by averaging the maximum and minimum thickness of a slab measured using calipers. This was done to accurately represent thickness of the slabs for the purpose of comparing their optical characteristics since some slabs gradually thickened or thinned toward the edges while some slabs remained completely flat toward the edges..

Transmission electron microscope (TEM) images of mesoporous silica slabs were taken using a $200 \mathrm{kV}$ FEI Tecnai TF20 TEM equipped with a field emission gun. The samples were first grounded, then suspended in ethanol and sonicated for $3 \mathrm{~min}$, and applied onto a 400-mesh carbon- 
coated copper TEM grid. The resulting TEM specimens were air dried before TEM analysis. Diameters of at least 50 distinct silica nanoparticles were manually measured using $\operatorname{ImageJ}^{1}$ software to calculate average diameter and standard deviation of the silica nanoparticles.

Low-temperature nitrogen adsorption-desorption isotherms were measured at $-196{ }^{\circ} \mathrm{C}$ using a surface area and porosity analyzer TriStar II 3020 (Micromeritics Instrument Corp., Norcross, GA, USA). Each sample was degassed in vacuum at $150-200{ }^{\circ} \mathrm{C}$ for $20-24 \mathrm{~h}$ prior to measurements. The specific surface area $S_{B E T}$ (in $\mathrm{m}^{2} \mathrm{~g}^{-1}$ ) was calculated using the BrunauerEmmett-Teller method $^{2}$ (BET) based on low-temperature nitrogen adsorption data in the relative pressure range $p / p_{0}=0.05-0.2$ and assuming the cross-sectional area of a nitrogen molecule to be $0.162 \mathrm{~nm}^{2}{ }^{3}$ The total specific pore volume $V_{t}$ (in $\mathrm{cm}^{3} \mathrm{~g}^{-1}$ ) was calculated by converting the number of moles of nitrogen adsorbed at a relative pressure $p / p_{0}=0.98$ to the volume of liquid nitrogen based on the liquid nitrogen molar density of $34.38 \mathrm{~cm}^{3} \mathrm{~mol}^{-1}$. Then, the porosity $\phi$ was calculated from the total specific pore volume $V_{t}$ according to ${ }^{5}$

$$
\phi=\frac{V_{t} \rho_{\mathrm{SiO}_{2}}}{1+V_{t} \rho_{\mathrm{SiO}_{2}}}
$$

The micropore volume $V_{m i}$ (in $\mathrm{cm}^{3} \mathrm{~g}^{-1}$ ) was calculated using the $\alpha_{s}$-comparative method ${ }^{5}$ in the reduced adsorption range $\alpha_{s}=0.4-0.8$ and using macroporous silica LiChrospher Si-1000 as a reference. ${ }^{6}$ The reduced adsorption $\alpha_{s}$ was defined as ${ }^{5}$

$$
\alpha_{s}=a_{r e f} / a_{r e f, 0.4}
$$

where $a_{r e f}$ and $a_{r e f, 0.4}$ are the volume of nitrogen adsorbed on the reference material surface at a given relative pressure $p / p_{0}$ and at $p / p_{0}=0.4$, respectively. In addition, the peak pore width $w_{p}$ (in $\mathrm{nm}$ ) was estimated from the position of peak maximum in the pore size distribution determined using the Kruk-Jaroniec-Sayari (KJS) method $^{7}$ based on the Barrett-Joyner-Halenda (BJH) $\operatorname{method}^{8}$ using (i) the adsorption branch of nitrogen isotherm, (ii) the modified Kelvin equation ${ }^{7}$ 
calibrated for cylindrical pores up to $19 \mathrm{~nm}$ in diameter, and (iii) the statistical film thickness curve derived from the nitrogen adsorption isotherm measured for macroporous silica LiChrospher Si$1000 .^{6}$

Two-dimensional small-angle X-ray scattering data were collected at the Stanford Synchrotron Lightsource (SSRL) using beamlines 1-5 with a wavelength of $0.1033 \mathrm{~nm}$ operated at an X-Ray energy of $12.002 \mathrm{keV}$ and detector distance of $2.870 \mathrm{~m}$ using a Rayonix-165 CCD detector. The data was then calibrated and reduced using the Nika package from Igor Pro. ${ }^{9}$ The reduced smallangle X-ray scattering (SAXS) patterns were fit using (a) unified model ${ }^{10,11}$ implementing correction to the Porod scaling factor ${ }^{12}$ and (b) pseudo-Voigt peaks by minimizing average relative error

$$
\left(\frac{\Delta I}{I}\right)_{\text {ave }}=\frac{1}{N} \sum_{i=1}^{N} \frac{\left|I_{\text {exp }, i}-I_{f i t, i}\right|}{I_{\text {exp }, i}}
$$

Pauly et al. ${ }^{13}$ showed that worm-like mesopores can yield up to two weak correlation peaks while remaining disordered. The contribution of these weak correlation peaks was subtracted from SAXS data to accurately calculate slopes of Porod plots.

Optical Characterization. The spectral normal-hemispherical transmittance $T_{n h, \lambda}$ and spectral diffuse transmittance $T_{d, \lambda}$ of the mesoporous silica slabs, at wavelength between 400 and $800 \mathrm{~nm}$, were measured with a double-beam UV-Vis spectrometer 3101-PC (Shimadzu, Kyoto, Japan) equipped with an integrating sphere ISR 3100 (Shimadzu, Kyoto, Japan) with internal diameter of $6 \mathrm{~cm}$. The measured transmitted signal $S_{n h, \lambda}$ was corrected for the reference signal $B_{n h, \lambda}$ and the dark signal $D_{n h, \lambda}$ according to

$$
T_{n h, \lambda}=\frac{s_{n h, \lambda}-D_{n h, \lambda}}{B_{n h, \lambda}-D_{n h, \lambda}} .
$$


The dark signal $D_{n h, \lambda}$ was collected in the absence of light while the reference signal $B_{n h, \lambda}$ was measured using the same procedure as that used to measure $S_{n h, \lambda}$ but in absence of sample. In addition, the diffuse transmitted signal $S_{d, \lambda}$ was measured by removing the cover of the back port of the integrating sphere opposite to the sample port so that the normal-normal beam of light left the integrating sphere while the scattered light transmitted through the sample was collected and measured. Moreover, haze $h_{\lambda}$ was estimated according to ASTM D1003-13 standard as ${ }^{14}$

$$
h_{\lambda}=\frac{S_{d, \lambda}}{S_{n h, \lambda}}-\frac{M_{d, \lambda}}{B_{n h, \lambda}} .
$$

where $M_{d, \lambda}$ was the signal measured using the same procedure as that used to measure $S_{d, \lambda}$ but without any sample present.

The color rendering index $C R I$ of the slabs quantifies the quality of color transmission through semitransparent materials. It ranges from 0 to 100 , with $C R I \approx 100$ indicating excellent color reproduction and $C R I \geq 95$ being acceptable. ${ }^{15}$ Most glass and windows have color rendering index $C R I=80-96 .{ }^{16}$ The CRI of mesoporous silica slabs was calculated according to BS EN 410:2011 standard ${ }^{17}$ taking the CIE (International Commission on Illumination) illuminant $D_{65}$ as the light source. First, the trichromatic coordinates $W_{i}^{*}, u_{i}^{*}$, and $v_{i}^{*}$, in the CIE 1964 uniform color space domain, of eight test colors were calculated from the spectral normal-hemispherical transmittance $T_{n h, \lambda}$. Then, the geometrical distance $\Delta E_{i}$, in the CIE 1964 uniform chromaticity space, between the color perceived with and without a sample was calculated for each of the eight test colors as ${ }^{17}$

$$
\Delta E_{i}=\sqrt{\Delta W_{i}^{* 2}+\Delta u_{i}^{* 2}+\Delta v_{i}^{* 2}} \quad \text { with } i=1,2, \ldots, 8 .
$$

Here, the trichromatic coordinates $W_{i}^{*}, u_{i}^{*}$, and $v_{i}^{*}$ of the reference illuminant $D_{65}$ were taken from BS EN 410:2011 standard. ${ }^{17}$ Finally, the color rendering index $C R I$ was calculated as ${ }^{17}$ 


$$
C R I=\frac{1}{8} \sum_{i=1}^{8}\left(100-4.6 \Delta E_{i}\right)
$$

The average $R_{a}$ and root mean squared $R_{R M S}$ surface roughness values of the mesoporous silica slabs were measured using a surface profilometer Dektak 6 (Veeco, Plainview, NY, USA) according to ${ }^{18}$

$$
R_{a}=\frac{1}{N} \sum_{i=1}^{N}\left|\bar{z}-z_{i}\right| \text { and } R_{R M S}=\sqrt{\frac{1}{N} \sum_{i=1}^{N}\left(\bar{z}-z_{i}\right)^{2}}
$$

where $N$ is the total number of vertical stylus displacement measurements, $z_{i}$ is the vertical stylus displacement at the $i^{\text {th }}$ equally spaced location along a line trace on the sample's surface, and $\bar{z}$ is the average vertical stylus displacement.

Thermal Characterization. Effective thermal conductivity $k_{e f f}$ of the mesoporous slabs was measured using the time-domain thermoreflectance (TDTR) method. ${ }^{19-21}$ It was calculated from the measured effective thermal effusivity $e_{e f f}$ of mesoporous silica slabs as ${ }^{19}$

$$
k_{e f f}=\frac{e_{e f f}^{2}}{c_{v, e f f}}=\frac{e_{e f f}^{2}}{\rho_{e f f} c_{p, e f f}}=\frac{e_{e f f}^{2}}{\phi_{\mathrm{SiO}_{2}} \rho_{\mathrm{SiO}_{2}} c_{p, e f f}},
$$

where $c_{v, e f f}$ is the effective volumetric heat capacity (in $\mathrm{J} \mathrm{m}^{3} \mathrm{~K}^{-1}$ ) where $\rho_{\text {eff }}, c_{p, e f f}$, and $\phi_{\mathrm{SiO}_{2}}=$ $1-\phi$ are the effective density (in $\mathrm{kg} \mathrm{m}^{-3}$ ), effective specific heat capacity (in $\mathrm{J} \mathrm{kg}^{-1} \mathrm{~K}^{-1}$ ), and silica volume fraction of the mesoporous silica slabs, respectively. First, the sample was coated with a 80 -nm thick Al film. Second, the Al-coated sample was heated to $160{ }^{\circ} \mathrm{C}$ for 1 hour at a pressure $<1 \mathrm{~Pa}$ in a cryostat to remove all physisorbed water, and then allowed to cool to room temperature (note that only $\mathrm{SiO}_{2}$-PTFE-rt sample was degassed while $\mathrm{SiO}_{2}$-PFC-hc sample was measured as prepared). Third, 2-fs laser pulses with wavelengths of 400 and $800 \mathrm{~nm}$ were used to generate heat (pump beam) and to detect temperature (probe beam), respectively. The pump and probe laser spot were $20 \mu \mathrm{m}$ in diameter to average the reflected signal over a surface area much larger than the average cross-sectional area of a pore and to measure the effective thermal 
effusivity of the porous sample. Here, the metallic film was used (i) as a transducer converting the photon energy of the pump laser pulse into thermal energy and (ii) as a sensor whose reflectance of the probe beam depends on temperature. After heating with the pump beam pulse, the temporal temperature decay of the $\mathrm{Al}$ film was recorded with the probe beam based on a linear relationship between reflectivity and temperature of the metallic film. Finally, the transient temperature decay curve was fitted with a multilayer heat conduction model to obtain the effective thermal effusivity. ${ }^{20}$ Measurements were performed at least at five different locations across the sample and averaged.

The effective specific heat capacity $c_{p, e f f}$ of degassed $\mathrm{SiO}_{2}$-PTFE-rt sample at room temperature was estimated by accounting for the presence of hydroxyl groups at the pore walls according to ${ }^{22}$

$$
c_{p, e f f}=c_{p, S i O_{2}}+M_{O H} \sigma_{O H} S_{B E T}\left(c_{p, O H}-c_{p, S i O_{2}}\right),
$$

where $c_{p, S i O_{2}}=0.733 \mathrm{~J} \mathrm{~g}^{-1} \mathrm{~K}^{-1}$ is the specific heat capacity of bulk amorphous silica, ${ }^{23} M_{O H}=17$ $\mathrm{g} \mathrm{mol}^{-1}$ is the molar mass of $\mathrm{OH}$ groups, $\sigma_{O H}=4.9 \mathrm{OH} \mathrm{nm}^{-2}$ is the surface density of $\mathrm{OH}$ groups, ${ }^{24}$ and $c_{p, O H}=1.58 \mathrm{~J} \mathrm{~g}^{-1} \mathrm{~K}^{-1}$ is the specific heat capacity of $\mathrm{OH}$ groups. ${ }^{25}$ Note that silica has a strong affinity for water and mesoporous silica offers large pore volume and surface area for water to condense in the mesopores. Thus, the effective specific heat capacity $c_{p, e f f}^{*}$ of the $\mathrm{SiO}_{2}$-PFC-hc sample under ambient conditions with non-zero relative humidity was estimated as

$$
c_{p, e f f}^{*}=\left(1-w_{\mathrm{H}_{2} \mathrm{O}}\right) c_{p, e f f}+w_{\mathrm{H}_{2} \mathrm{O}} c_{p, \mathrm{H}_{2} \mathrm{O}},
$$

where $w_{H_{2} O}=0.05$ is the estimated weight fraction of water adsorbed on the slab surface, $c_{p, e f f}$ is the effective specific heat capacity of degassed mesoporous silica given by Equation (S10), and $c_{p, \mathrm{H}_{2} \mathrm{O}}=4.181 \mathrm{~J} \mathrm{~g}^{-1} \mathrm{~K}^{-1}$ is the specific heat capacity of water at $25^{\circ} \mathrm{C} .{ }^{26}$ 
The combined relative uncertainty at $95 \%$ confidence level of the effective thermal conductivity was calculated as

$$
\frac{\Delta k_{e f f}}{k_{e f f}}=\sqrt{\left(\frac{\Delta e_{e f f}^{2}}{e_{e f f}^{2}}\right)^{2}+\left(\frac{\Delta \phi_{S i O_{2}}}{\phi_{S i O_{2}}}\right)^{2}+\left(\frac{\Delta c_{p, e f f}}{c_{p, e f f}}\right)^{2}},
$$

where $\Delta e_{\text {eff }}^{2}$ was estimated as a standard deviation of a mean value at $95 \%$ confidence level and $\Delta \phi_{S i O_{2}}=\Delta \phi= \pm 0.02$ was estimated based on prior experience. Uncertainty of the effective specific heat capacity was estimated as

$$
\begin{gathered}
\Delta c_{p, e f f}=M_{O H} \sigma_{O H}\left(c_{p, O H}-c_{p, S i O_{2}}\right) \Delta S_{B E T} \text { and } \\
\Delta c_{p, e f f}^{*}=\sqrt{\left[\left(1-w_{\mathrm{H}_{2} O}\right) \Delta c_{p, e f f}\right]^{2}+\left[\left(c_{p, H_{2} O}-c_{p, e f f}\right) \Delta w_{H_{2} O}\right]^{2}}
\end{gathered}
$$

where $\Delta S_{B E T}=0.1 S_{B E T} .{ }^{22}$

Mechanical Characterization. Effective Young's modulus $E_{\text {eff }}$ and hardness $H_{\text {eff }}$ of the mesoporous silica slabs were measured using a MTS Nanoindenter XP instrument (MTS Nano Instruments Inc., Oak Ridge, TN, USA) equipped with a diamond Berkovich pyramidal tip. The continuous stiffness measurement (CSM) method was used to measure Young's modulus and hardness of the mesoporous slabs as a function of displacement that were then averaged over the displacement range 500-2000 $\mathrm{nm}$. Each sample was indented at 16 different locations arranged in a $4 \times 4$ grid with $25 \mu \mathrm{m}$ wide pitch. The sample-average Young's modulus and hardness were calculated by averaging all valid indentation-average values. All samples were indented using the harmonic displacement of $2 \mathrm{~nm}$, frequency of $45 \mathrm{~Hz}$, strain rate of $0.05 \mathrm{sec}^{-1}$, and depth limit of $2000 \mathrm{~nm}$. Poisson's ratio was taken as $0.20 .27-29$ 

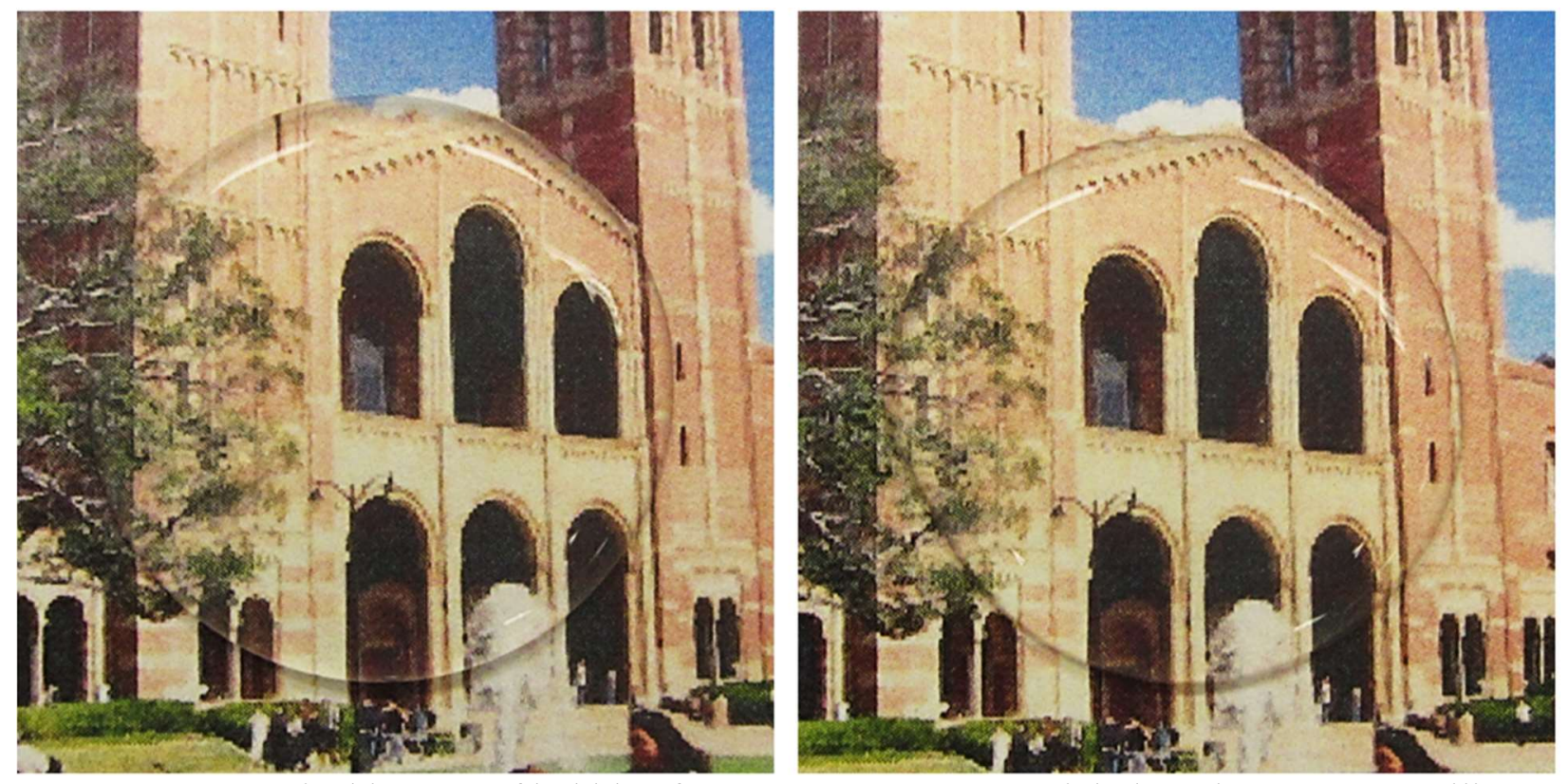

Figure S1. Optical images of bubbles-free transparent nanoparticle-based mesoporous silica monolithic slabs prepared on defect-free PTFE at room temperature. Both slabs had diameter of $\approx 2.5 \mathrm{~cm}$ and average thickness $\approx 2 \mathrm{~mm}$. 


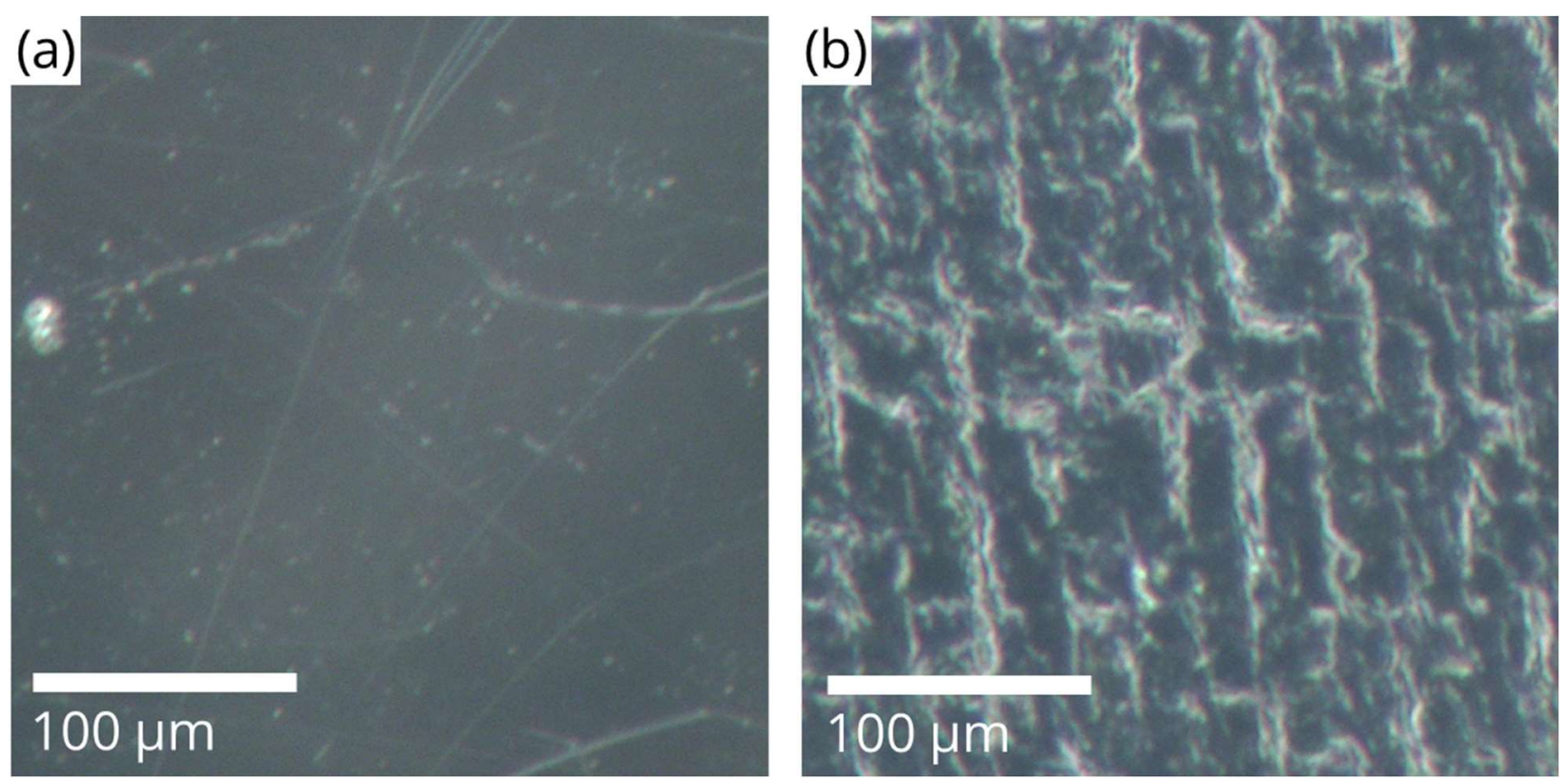

Figure S2. Optical images of (a) bottom surface of a $\mathrm{SiO}_{2}-\mathrm{PFC}$ slab with smooth surface from the PFC liquid (few visible scratches and defects are due to handling of the slab) and (b) bottom surface of a $\mathrm{SiO}_{2}$-PTFE slab with noticeable surface roughness from the PTFE mold. 

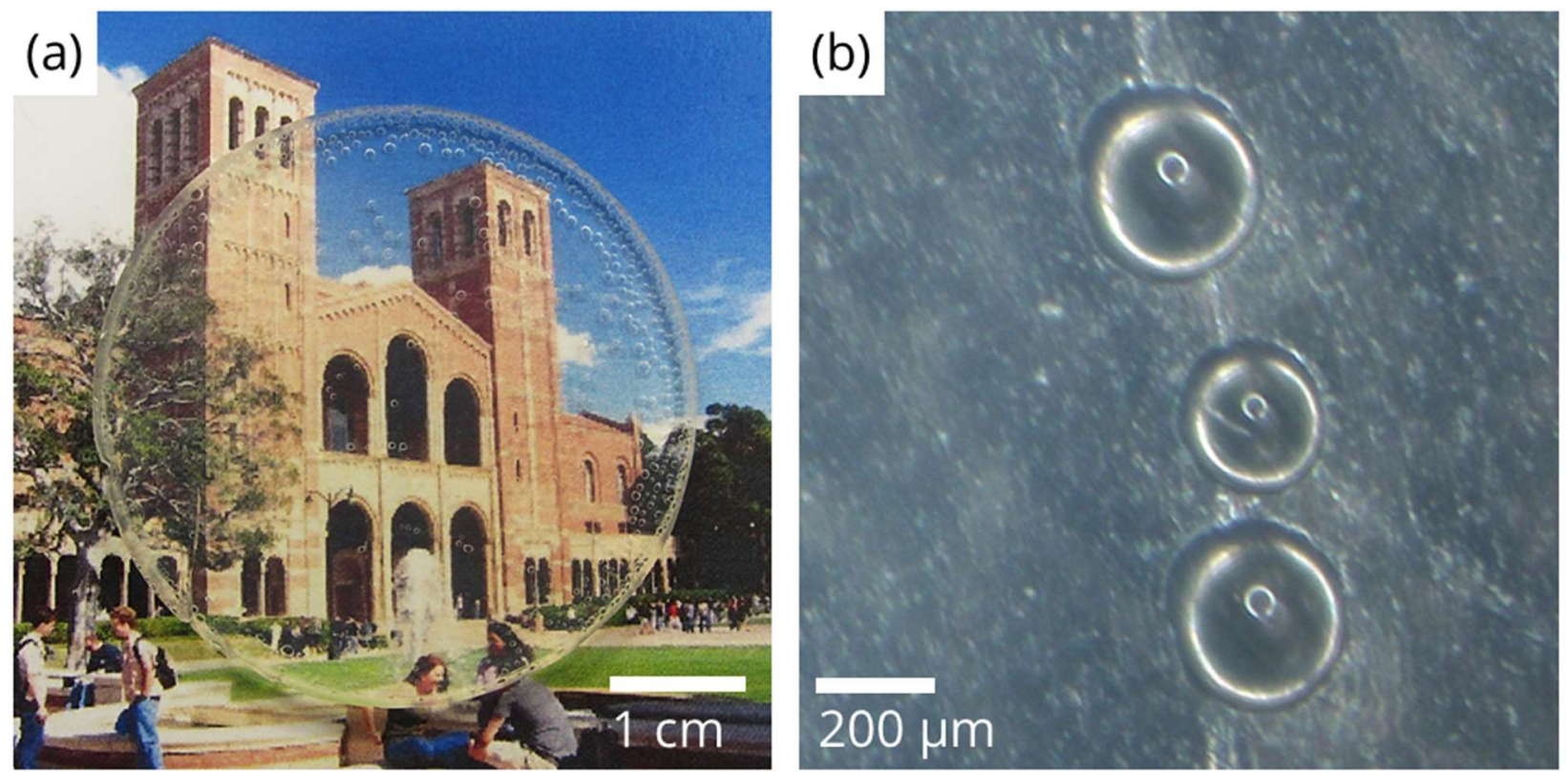

Figure S3. Optical images of (a) nanoparticle-based mesoporous silica monolithic slab with bubbles on the bottom surface prepared on PTFE at room temperature (diameter $4.5 \mathrm{~cm}$ and average thickness $3.5 \mathrm{~mm}$ ) and (b) bubbles at the bottom of a $\mathrm{SiO}_{2}-\mathrm{PTFE}-\mathrm{rt}$ slab formed along a scratch in PTFE (also imprinted in the slab). 


\section{References}

(1) Schneider, C. A.; Rasband, W. S.; Eliceiri, K. W. NIH Image to ImageJ: 25 Years of Image Analysis. Nat. Methods 2012, 9, 671-675. https://doi.org/10.1038/nmeth.2089.

(2) Brunauer, S.; Emmett, P. H.; Teller, E. Adsorption of Gases in Multimolecular Layers. $J$. Am. Chem. Soc. 1938, 60 (2), 309-319. https://doi.org/10.1021/ja01269a023.

(3) Kruk, M.; Jaroniec, M. Gas Adsorption Characterization of Ordered Organic-Inorganic Nanocomposite Materials. Chem. Mater. 2001, 13 (10), 3169-3183. https://doi.org/10.1021/cm0101069.

(4) Naono, H.; Hakuman, M.; Shiono, T. Analysis of Nitrogen Adsorption Isotherms for a Series of Porous Silicas with Uniform and Cylindrical Pores: A New Method of Calculating Pore Size Distribution of Pore Radius 1-2 Nm. J. Colloid Interface Sci. 1997, 186 (2), 360368. https://doi.org/10.1006/jcis.1996.4677.

(5) Rouquerol, J.; Rouquerol, F.; Llewellyn, P.; Maurin, G.; Sing, K. S. W. Adsorption by Powders and Porous Solids: Principles, Methodology and Applications, 2 edition.; Academic Press: Amsterdam, 2013.

(6) Jaroniec, M.; Kruk, M.; Olivier, J. P. Standard Nitrogen Adsorption Data for Characterization of Nanoporous Silicas. Langmuir 1999, 15 (16), 5410-5413. https://doi.org/10.1021/la990136e.

(7) Kruk, M.; Jaroniec, M.; Sayari, A. Application of Large Pore MCM-41 Molecular Sieves To Improve Pore Size Analysis Using Nitrogen Adsorption Measurements. Langmuir 1997, 13 (23), 6267-6273. https://doi.org/10.1021/la970776m.

(8) Barrett, E. P.; Joyner, L. G.; Halenda, P. P. The Determination of Pore Volume and Area Distributions in Porous Substances. I. Computations from Nitrogen Isotherms. J. Am. Chem. Soc. 1951, 73 (1), 373-380. https://doi.org/10.1021/ja01145a126.

(9) Ilavsky, J. Nika: Software for Two-Dimensional Data Reduction. J. Appl. Crystallogr. 2012, 45 (2), 324-328. https://doi.org/10.1107/S0021889812004037.

(10) Beaucage, G. Approximations Leading to a Unified Exponential/Power-Law Approach to Small-Angle Scattering. J. Appl. Crystallogr. 1995, 28 (6), 717-728. https://doi.org/10.1107/S0021889895005292.

(11) Beaucage, G. Small-Angle Scattering from Polymeric Mass Fractals of Arbitrary MassFractal Dimension. J. Appl. Crystallogr. 1996, 29 (2), 134-146. https://doi.org/10.1107/S0021889895011605.

(12) Hammouda, B. Analysis of the Beaucage Model. J. Appl. Crystallogr. 2010, 43 (6), 14741478. https://doi.org/10.1107/S0021889810033856.

(13) Pauly, T. R.; Liu, Y.; Pinnavaia, T. J.; Billinge, S. J. L.; Rieker, T. P. Textural Mesoporosity and the Catalytic Activity of Mesoporous Molecular Sieves with Wormhole Framework Structures. J. Am. Chem. Soc. 1999, 121 (38), 8835-8842. https://doi.org/10.1021/ja991400t.

(14) Standard Test Method for Haze and Luminous Transmittance of Transparent Plastics (ASTM D1003-13). ASTM International.

(15) Gunde, M. K.; Krašovec, U. O.; Platzer, W. J. Color Rendering Properties of Interior Lighting Influenced by a Switchable Window. JOSA A 2005, 22 (3), 416-423. https://doi.org/10.1364/JOSAA.22.000416.

(16) Dangol, R.; Kruisselbrink, T.; Rosemann, A. Effect of Window Glazing on Colour Quality of Transmitted Daylight. J. Daylighting 2017, 4 (2), 37-47. https://doi.org/10.15627/jd.2017.6. 
(17) Glass in Building. Determination of Luminous and Solar Characteristics of Glazing (BS EN 410:2011 ). British Standards Institution.

(18) Den Outer, A.; Kaashoek, J. F.; Hack, H. R. G. K. Difficulties with Using Continuous Fractal Theory for Discontinuity Surfaces. Int. J. Rock Mech. Min. Sci. Geomech. Abstr. 1995, 32 (1), 3-9. https://doi.org/10.1016/0148-9062(94)00025-X.

(19) Hu, Y.; Zeng, L.; Minnich, A. J.; Dresselhaus, M. S.; Chen, G. Spectral Mapping of Thermal Conductivity through Nanoscale Ballistic Transport. Nat. Nanotechnol. 2015, 10 (8), 701706. https://doi.org/10.1038/nnano.2015.109.

(20) Cahill, D. G. Analysis of Heat Flow in Layered Structures for Time-Domain Thermoreflectance. Rev. Sci. Instrum. 2004, 75 (12), 5119-5122. https://doi.org/10.1063/1.1819431.

(21) Schmidt, A. J.; Chen, X.; Chen, G. Pulse Accumulation, Radial Heat Conduction, and Anisotropic Thermal Conductivity in Pump-Probe Transient Thermoreflectance. Rev. Sci. Instrum. 2008, 79 (11), 114902. https://doi.org/10.1063/1.3006335.

(22) Marszewski, M.; Butts, D.; Lan, E.; Yan, Y.; King, S. C.; McNeil, P. E.; Galy, T.; Dunn, B.; Tolbert, S. H.; Hu, Y.; et al. Effect of Surface Hydroxyl Groups on Heat Capacity of Mesoporous Silica. Appl. Phys. Lett. 2018, $112 \quad$ (20), 201903. https://doi.org/10.1063/1.5027080.

(23) Richet, P.; Bottinga, Y.; Denielou, L.; Petitet, J. P.; Tequi, C. Thermodynamic Properties of Quartz, Cristobalite and Amorphous SiO2: Drop Calorimetry Measurements between 1000 and $1800 \mathrm{~K}$ and a Review from 0 to $2000 \mathrm{~K}$. Geochim. Cosmochim. Acta 1982, 46 (12), 2639-2658. https://doi.org/10.1016/0016-7037(82)90383-0.

(24) Zhuravlev, L. T. Concentration of Hydroxyl Groups on the Surface of Amorphous Silicas. Langmuir 1987, 3 (3), 316-318. https://doi.org/10.1021/la00075a004.

(25) Rihani, D. N.; Doraiswamy, L. K. Estimation of Heat Capacity of Organic Compounds from Group Contributions. Ind. Eng. Chem. Fundam. 1965, 4 (1), 17-21. https://doi.org/10.1021/i160013a003.

(26) CRC Handbook of Chemistry and Physics, 98th edition 2017-2018.; Rumble, J. R., Ed.; CRC Press, Taylor \& Francis Group: Boca Raton London New York, 2017.

(27) Gross, J.; Reichenauer, G.; Fricke, J. Mechanical Properties of SiO 2 Aerogels. J. Phys. Appl. Phys. 1988, 21 (9), 1447. https://doi.org/10.1088/0022-3727/21/9/020.

(28) Moner-Girona, M.; Roig, A.; Molins, E.; Martínez, E.; Esteve, J. Micromechanical Properties of Silica Aerogels. Appl. Phys. Lett. 1999, 75 (5), 653-655. https://doi.org/10.1063/1.124471.

(29) Patil, S. P.; Rege, A.; Sagardas; Itskov, M.; Markert, B. Mechanics of Nanostructured Porous Silica Aerogel Resulting from Molecular Dynamics Simulations. J. Phys. Chem. B 2017, 121 (22), 5660-5668. https://doi.org/10.1021/acs.jpcb.7b03184. 